\title{
6 DOF pose estimation of polyhedral objects based on analysis of geometric features in $x$-ray images
}

\author{
Jae Wan Kim, Young Jun Roh and Hyung Suck. Cho* \\ Department of Mechanical Engineering, Korea Advanced Institute of Science and Technology
}

\begin{abstract}
An x-ray vision can be a unique method to monitor in real time and analyze the motion of mechanical parts which are invisible from outside. Our problem is to identify the pose, i.e. the position and orientation of an object from x-ray projection images. It is assumed here that the x-ray imaging conditions that include the relative coordinates of the x-ray source and the image plane are predetermined and the object geometry is known. In this situation, an x-ray image of an object at a given pose can be estimated computationally by using a priori known x-ray projection image model. It is based on the assumption that a pose of an object can be determined uniquely to a given $\mathrm{x}$-ray projection image. Thus, once we have the numerical model of x-ray imaging process, x-ray image of the known object at any pose could be estimated. Then, among these estimated images, the best matched image could be searched and found. When adequate features in the images are available instead of the image itself, the problem becomes easier and simpler. In this work, for simplicity, only polyhedral objects are considered whose image features consist of corner points and edge lines in their projection images. Based on the corner points and lines found in the images, the best-matched pose of a polyhedral object can be determined. To achieve this, we propose an adequate and efficient image processing algorithm to extract the features of objects in x-ray images. The performance of the algorithms is discussed in detail including the limitations of the method. To evaluate the performance of the proposed method a series of simulation studies is carried out for various imaging conditions.
\end{abstract}

Keywords : Pose estimation, x-ray image, feature extraction.

\section{INTRODUCTION}

Pose estimation of a three dimensional (3D) object has many applications in industrial problems such as process monitoring and control, assembly and PCB inspection. Especially, pose estimation from a single image with respect to a known object is a classical problem and has been widely studied in robot vision. The techniques of pose estimation from 2D images (the 3D/2D matching problem) can be classified into two general categories as the matching primitives used. The first one is based on the features identified on the surface of the object [1]. In this technique, the closed-form solution is available only in the case of four or less feature points. If there are more than four correspondences, closed-form solution does not exist and should be solved by iterative non-linear solutions. The second one is based on the extreme contours of the object on the image. In this technique, the surface of an object must be modeled as a set of pre-determined patches or mathematical functions. Lowe proposed an iterative approach for aligning the projected extreme contours with edges found in the image [1][2]. However, some limitations and problems in pose estimation using a conventional camera image has been pointed out, and they are listed as follows :

a) Surface reflection problem :

A camera image is affected by surface conditions because camera senses light reflected from the surface of an object. Especially, in case of specular surface objects, it is very difficult to analyze the image due to the highlight spots in the images.

b) Occlusion problem :

We cannot acquire information on the occluded region or inner part of certain structure through camera images, but only the surface information is available.

\footnotetext{
* Correspondence : Email : hscho@lca.kaist.ac.kr ; WWW : http://lca.kaist.ac.kr ; Telephone : +82-42-869-3213 ; Fax : +82-42-869-
}

3210; ME3213, Dept. of Mechanical Eng., KAIST, 373-1, Kusong-dong, Yusong-gu, Daejon, Korea, 305-701. 
In practice, there are many mechanical parts of which the surface is specular, or sometimes they are occluded by the other parts and invisible from outside. Then, camera vision system is not so much an efficient and reliable method to inspect or measure them. In these situations, x-ray system can be a good solution to overcome these limitations due to its penetrating characteristics. These days, the need to X-ray imaging method is increasing in industrial field such as solder joint inspection system in PCB board and the battery of cellular phone inspection system. Until now, it has been mainly used in inspecting defects of products. However, it also can be a good method to measure the shape or pose of an object. There have been several applications of pose estimation using x-ray image in medical research. For pose estimation of artificial knee implants in fluoroscopy images, Hoff and Komistek et. al. [3] propose a template matching technique for the 2D/2D matching. In this technique, the library of images is created in advance, which consists of views of the 3D models for the implant components rendered at different rotations. And the pose of the implant is estimated by matching a fluoroscopy image to the library. This technique needs a large size of library and may take a long matching time. Another approach using contours for the 3D/2D matching was proposed by Lavallee and Szeliski [1] to estimate the pose of free-form objects such as vertebra for spine surgery or skull for neurosurgery. Here, a free-form 3D object is modeled in advance by MRI method and a contour in X-ray image is matched to that of the 3D model. In addition to this medical application, the problem of pose estimation can also be used to measure and monitor a dynamic motion of occluded components and to calibrate motional parts in a system in industrial field. Until now, x-ray method has mainly been mainly used for the inspection but not for the measurements in industrial field. Therefore, the measurement problems need to be investigated in more detail by using x-ray system.

In this paper, we have investigated on a pose, a position and orientation of an object, estimation problem with single x-ray image. Here, it is assumed that the x-ray imaging conditions that include the relative coordinates of the x-ray source and the image plane are predetermined and the object geometry is known. For simplicity, only polyhedral objects are considered whose image features consist of corner points and edge lines in their projection images. Based on the corner points and lines found in the images, the best-matched pose of a polyhedral object can be determined. To achieve this, we propose an adequate and efficient image processing algorithm to extract the features of objects in x-ray images. The performance of the algorithms is discussed in detail including the limitations of the method. To evaluate the performance of the proposed method a series of simulation studies are carried out and discussed.

\section{X-RAY IMAGING SYSTEM}

Figure 1(a) represents an x-ray imaging system for our investigation, which is composed of the $\mathrm{x}$-ray tube, $\mathrm{x}$-ray image device and a rotating and tilting stage to manipulating the object on it. The parameters and system coordinates are illustrated in figure 1 (b). The stage has 6 degree of freedom with 4 translations $(x, y, z, f)$, tilting and rotating motion $(\phi, \theta)$. In this configuration, the ratio between $f$ and $L$ becomes a magnification $m$ onto the image plane.

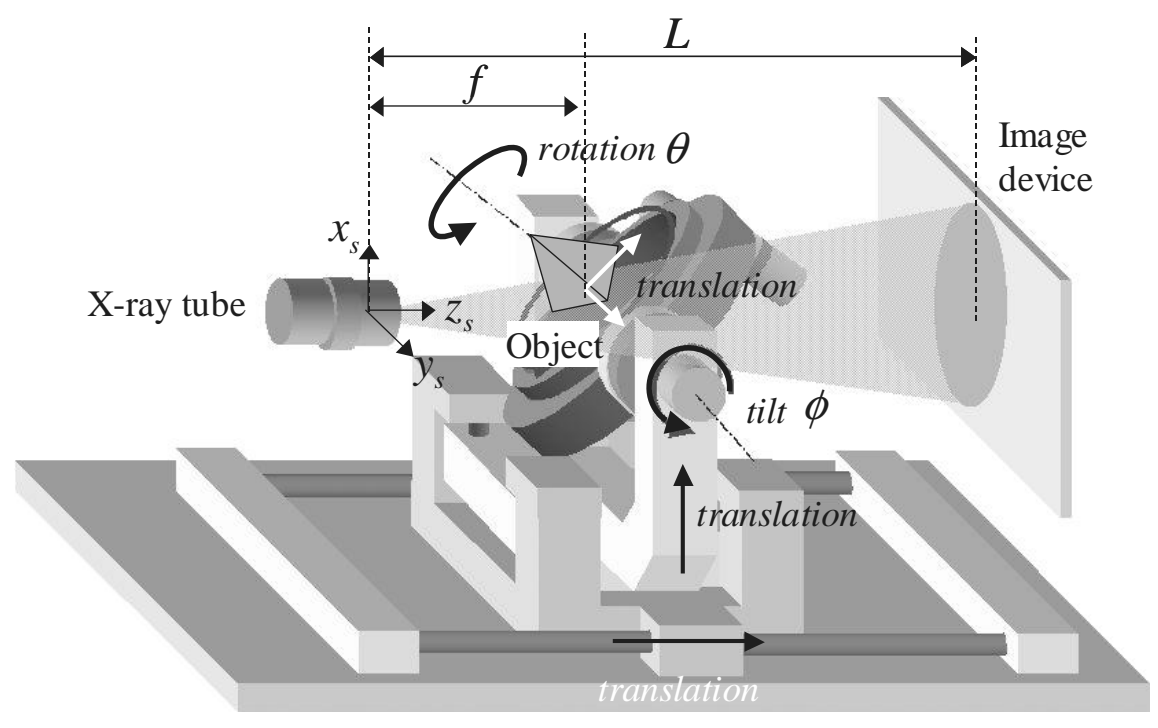

Figure 1. An x-ray imaging system and its coordinates. 

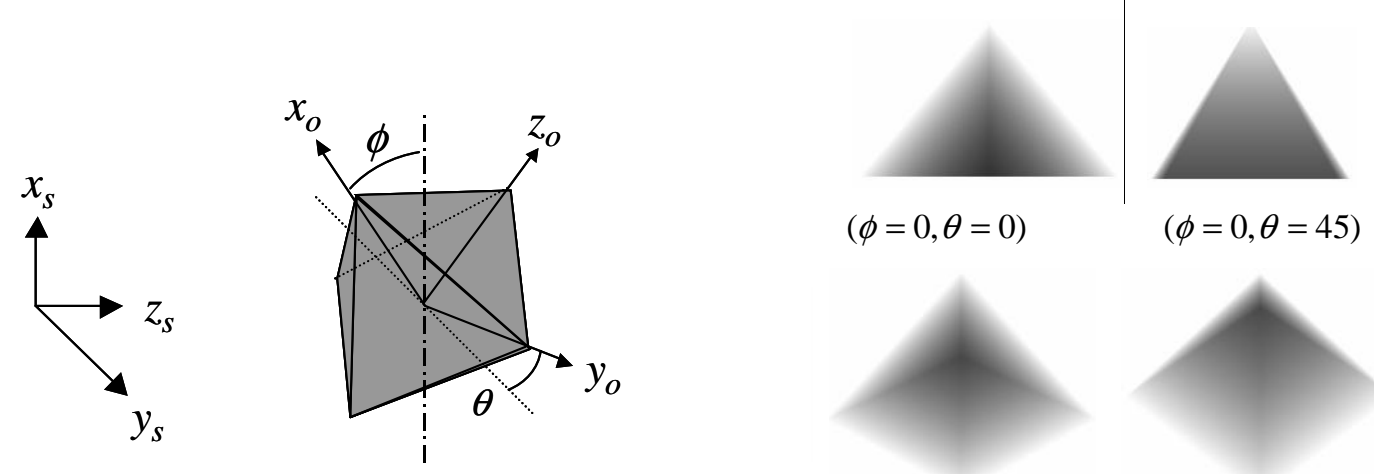

$(\phi=0, \theta=0)$
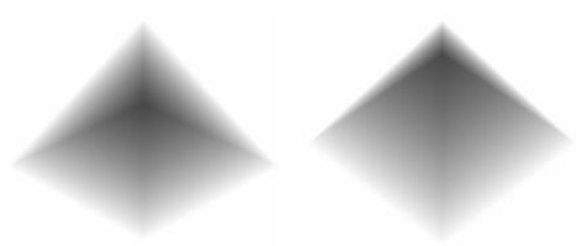

$(\phi=30, \theta=0)$

$(\phi=45, \theta=0)$

Figure 2. X-ray images of a pyramidal shape object with different views

Figure 2 shows the x-ray images of a pyramidal shape object from different views which are achieved by manipulating the stage on which the object lies. Here, $x, y, z$ and $x_{o}, y_{o}, z_{o}$ indicate the world coordinates and an object coordinates respectively.

\section{X-RAY IMAGE PROCESSING ALGORITHM}

Since the pose estimation in this work is based on the features such as edges and corner points in the images, it is important to extract exact features. There are a lot of image processing technique to extract image features in computer vision. However, most of them are for conventional camera images and not applicable to x-ray images due to its inherent characteristics. This comes from the different imaging principles : images in camera vision are made by the reflected lights on the surface of an object, on the other hand x-ray images are made using transmitted and decayed ray through an object. In $\mathrm{x}$-ray imaging method, x-ray intensity decays exponentially when it is passing through an object. The amount of decay depends on initial intensity, penetrating length of x-ray and material properties such as density as represented in the following equation :

$$
I=I_{0} \cdot e^{-\mu x}
$$

In this equation, $I$ and $I_{o}$, respectively, are the intensity of x-ray before and after the penetration and $\mu$ is a decay constant of $\mathrm{X}$-ray, and $x$ is the penetrating length[4][5]. Thus, intensity change itself in an X-ray image does not directly indicate edge information as in camera images. Also, x-ray images generally include a lot of noises due to the scattered rays within the shield cabinet. In this section, we propose an efficient image processing method to extract the features in x-ray image considering those characteristics. Especially, since polyhedral objects are considered in this research, we focus on extracting edges and corner points as image features. The procedure of extracting edges and corner points in x-ray images is represented as a block diagram in figure 3 . Here, the line edges are found from a proposed plane fit method, then the corner points are determined using these lines, which are explained in the following sections.

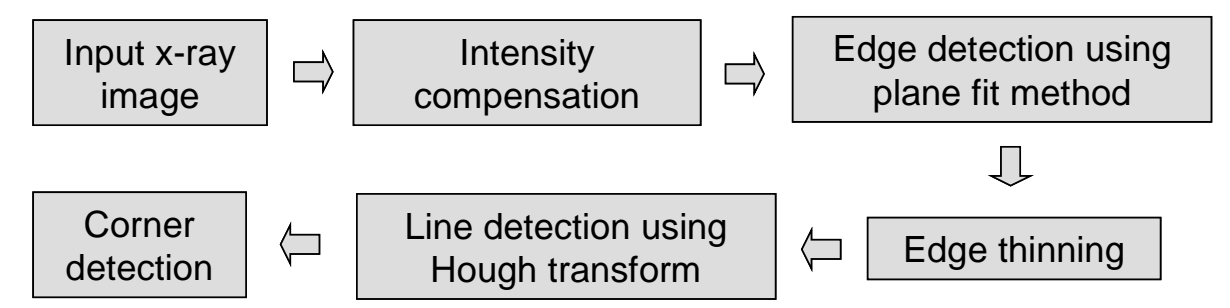

Figure 3. The procedure of the proposed edge detection method 


\subsection{Edge detection algorithm}

\subsubsection{Intensity linearization using log filter}

Intensity in an x-ray image can be thought as a function of penetrating length of the ray when we assume that the object is made of one material. Figure 4 (a) is a simulated x-ray image for a polyhedron, a pyramidal shape object. Since the ray transmits the longest length within the object in the peak points, the intensity for the ray in x-ray image is the lowest with decayed $x$-ray. Due to the exponentially decaying x-ray intensity as the penetrating length, the x-ray image of the pyramid object has an intensity distribution, which is represented by a topographic surface in figure 4 (c). Here, we can see that the surface of the intensity plot is distorted exponentially compared to the object surface. To detect the edges of a polygon in an x-ray image, this distorted intensity distribution needs to be linearized in advance. This is achieved by a log transform with normalization as in equation (2) and (3), which is a process of converting the exponentially decaying intensity function to a linearized one.

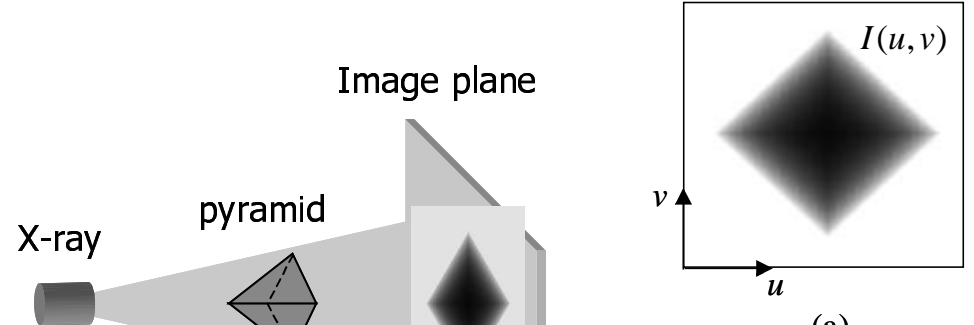

(a)

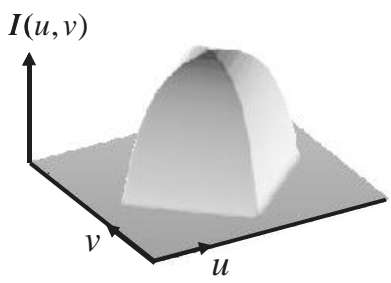

(c)

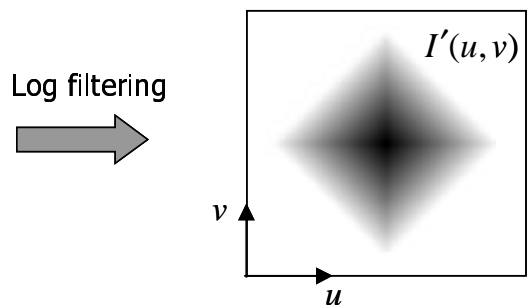

(b)

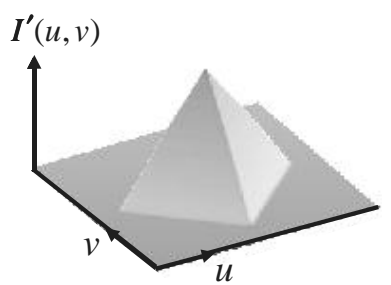

(d)

(a) X-ray image of pyramidal shape, (b) Log filtered image,

(c) Topographic surface of raw image, (d) Topographic surface of log filtered image.

Figure 4. Intensity compensation in x-ray image

$$
\begin{gathered}
L(u, v)=\log [I(u, v)] \\
I^{\prime}(u, v)=\frac{L(u, v)-\min [L(u, v)]}{\max [L(u, v)]-\min [L(u, v)]} \times\left(2^{n}-1\right)
\end{gathered}
$$

where $I(u, v)$ is the intensity of a pixel at $(u, v)$ in the image and $n$ is the intensity bit number. Figure 4 (d) shows a typical topographic surface plot of the log filtered image, which is a plot of intensities with respect to the image coordinates $(u, v)$. Since the edges of the object are represented straight lines in this linearized topographic surface, it makes easier to detect edges from this surface rather than from the original one.

\subsubsection{Edge detection by plane fit method}

In this research, the edges in an x-ray image of a polyhedral object are found by using the topographic surface of the log filtered image. There is an important characteristic in the topographic surface in case of a polyhedral object considered here. Here, the topographic surface represents the transmitting length of $x$-ray rays passing through an object, and it is also composed of polygonal facets when the object is a polyhedron. This can be easily understood by intuition. Thus, the edges in $\mathrm{x}$-ray image can be determined at the cross lines between the facets in the corresponding topographic surface. To achieve this, we propose a plane fit method that enhances the edges in x-ray images of polyhedrons. The topographic surface, here, is 
considered as a surface made of plane elements. Thus, if we try to find best fit planes with a small mask window over the topographic surface as illustrated in figure 5, the plane elements could be determined as approximated plane equations.
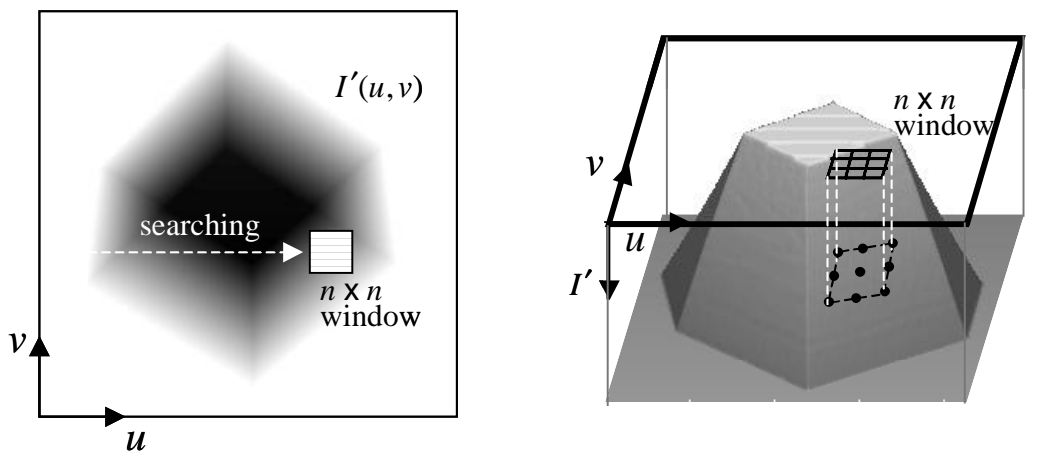

Figure 5. Plane fit method on topographic surface

Then, the edges are determined from this plane fit result, since the fitting result has large error at the edges where two or more planes are present. The plane fit within a mask window, which is denoted by equation (4), is achieved by a least square method.

$$
a u+b v+c=I^{\prime}
$$

where the parameters $a, b$ and $c$ are the constants of the plane equation and $I^{\prime}$ is intensity value of a point $(u, v)$. The fitting square error $E_{p}$ is then defined by equation (5) as

$$
E_{p}=\sum_{i=0}^{N-1}\left(I_{i}^{\prime}-a u_{i}-b v_{i}-c\right)^{2} .
$$

where $i$ and $I_{i}^{\prime}$ are an index of a pixel within the window and its intensity, respectively.

From the least square expansion, the coefficients of the best fit plane for the mask are determined by equation (6), which comes from the partial derivatives of $E_{p}$ with respect to the coefficients to be zero

$$
\left[\begin{array}{lll}
\sum_{i=0}^{N-1} u_{i}^{2} & \sum_{i=0}^{N-1} u_{i} v_{i} & \sum_{i=0}^{N-1} u_{i} \\
\sum_{i=0}^{N-1} u_{i} v_{i} & \sum_{i=0}^{N-1} v_{i}^{2} & \sum_{i=0}^{N-1} v_{i} \\
\sum_{i=0}^{N-1} u_{i} & \sum_{i=0}^{N-1} v_{i} & \sum_{i=0}^{N-1} 1
\end{array}\right]\left[\begin{array}{l}
a \\
b \\
c
\end{array}\right]=\left[\begin{array}{l}
\sum_{i=0}^{N-1} I_{i} u_{i} \\
\sum_{i=0}^{N-1} I_{i} v_{i} \\
\sum_{i=0}^{N-1} I_{i}
\end{array}\right] .
$$

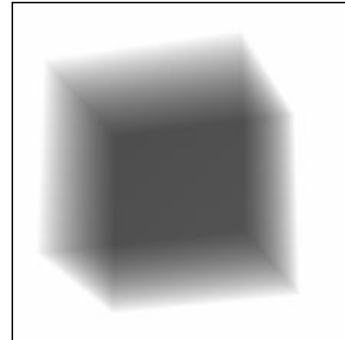

(a) Raw x-ray image

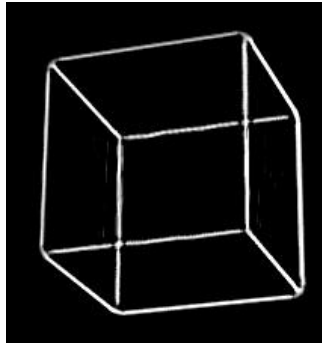

(b) Edge detection by plane fit method

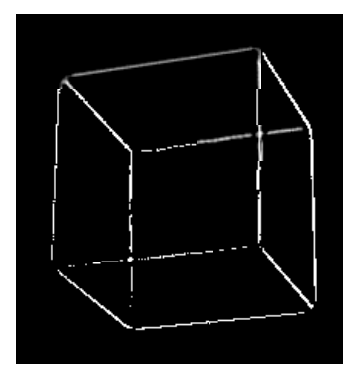

(c) Thinned edge image

Figure 6. An example of edge detection by plane fit method 
From the fitting results, we can obtain the square error defined in equation (5) at a point $(u, v)$, which means the strength of edge in topographic surface. Figure 6 shows an example of edge detection result by the proposed method. In practice, the edges obtained from the plane fit method are some thick and need to be thinned as shown in figure 6 (c).

\subsection{Corner points detection algorithm}

The corner points can be important features in an X-ray image of polyhedron objects. In this section, corner points detection algorithm in an edge image is proposed. Once an edge image of the given x-ray image is obtained from the proposed edge detection method, the straight lines in the edge image can be extracted through Hough transform method. Then, the corner points are determined at the points where three or more lines intersect. Figure 7 shows an example of extracting corner points for a cube.

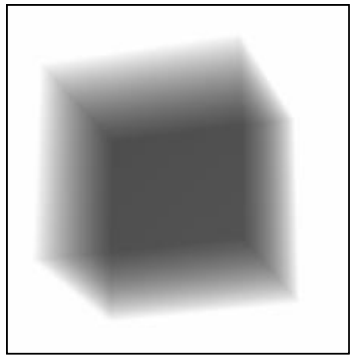

(a) Raw image

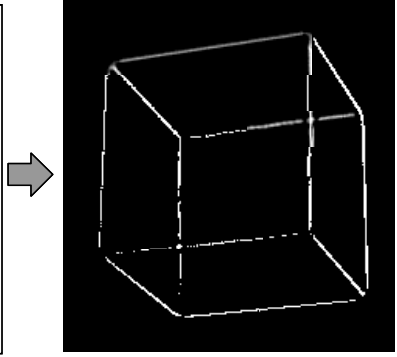

(b) Edge image

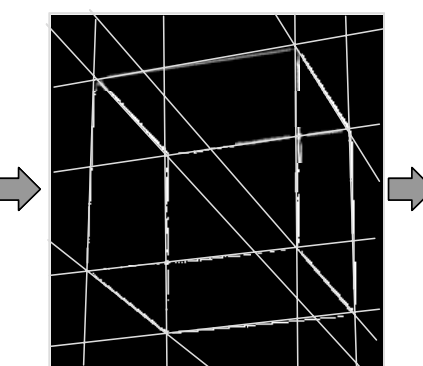

(c) Line extraction

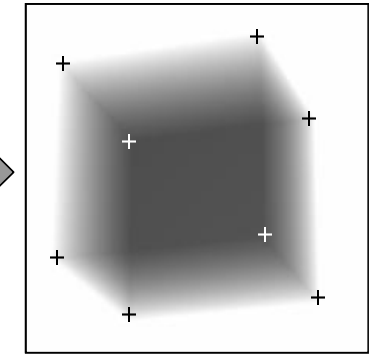

(d) Corner points detection

Figure 7. Corner points detection procedure.

Table 1 represents the errors of corner points in case of a cube for various values of the pose parameters, $f$ and $\theta$. From these simulations, average error of the detected corner points is about 0.8 pixel, sub-pixel error, in a 256 by 256 image.

Table 1. The error of corner points detected for of a cube

\begin{tabular}{|c|ccccc|c|}
\hline$\theta\left({ }^{\circ}\right)$ & 35 & 40 & 45 & 50 & 60 & average \\
\hline 15 & 0.75 & 0.76 & 0.75 & 0.77 & 0.90 & 0.78 \\
16 & 0.96 & 0.84 & 0.74 & 0.71 & 0.93 & 0.84 \\
17 & 0.55 & 0.67 & 0.75 & 0.84 & 0.89 & 0.74 \\
18 & 0.76 & 0.95 & 0.67 & 0.80 & 1.07 & 0.85 \\
19 & 0.93 & 0.79 & 0.71 & 0.77 & 1.12 & 0.86 \\
20 & 0.67 & 0.58 & 0.79 & 0.71 & 0.72 & 0.70 \\
21 & 0.71 & 0.72 & 0.82 & 0.71 & 0.84 & 0.76 \\
22 & 0.89 & 0.67 & 0.58 & 0.86 & 0.79 & 0.76 \\
23 & 0.86 & 0.96 & 0.58 & 0.87 & 0.75 & 0.80 \\
24 & 0.74 & 0.77 & 0.73 & 0.70 & 0.82 & 0.75 \\
25 & 0.77 & 0.95 & 0.89 & 0.49 & 0.66 & 0.75 \\
\hline average & 0.78 & 0.79 & 0.73 & 0.75 & 0.86 & 0.78 \\
\hline
\end{tabular}

\section{POSE ESTIMATION}

In this research, we aimed at estimating a pose of an object from an x-ray image using the image feature information, which are the corner points and edge lines in case of polyhedral objects. In general, it has been known that the pose of a three dimensional object is not determined uniquely by an analytical solution from just one image. Rather than that, the best matched pose for the given image can be solved iteratively based on the perspective imaging model.

\subsection{Perspective projection model}

Figure 8 illustrates a perspective projection model of an x-ray imaging system. In this work, a normalized virtual plane of which the distance from $\mathrm{x}$-ray source is unit length, is considered as an imaging plane for convenience as shown in the figure. 
Here, it is our aim to determine the relationships between the object coordinates and x-ray source coordinates, which is expressed by a coordinate transformation as :

$$
\left[\begin{array}{c}
x_{o} \\
y_{o} \\
z_{o} \\
1
\end{array}\right]=\left[\begin{array}{cc}
\mathbf{R} & \mathbf{T} \\
0 & 1
\end{array}\right]\left[\begin{array}{c}
x_{s} \\
y_{s} \\
z_{s} \\
1
\end{array}\right]
$$

where $\mathbf{R} \in \mathbb{R}^{3 \times 3}$ and $\mathbf{T} \in \mathbb{R}^{3 \times 1}$ are the rotation and translation matrices, respectively used for the coordinate transformation.

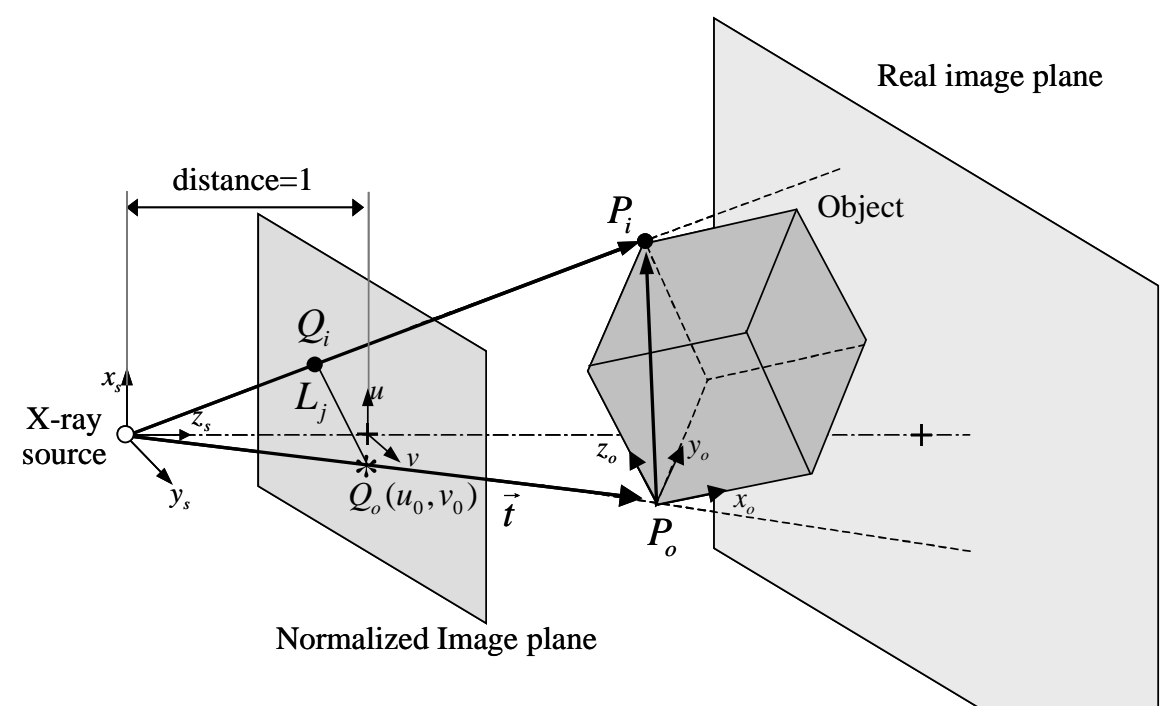

Figure 8. The perspective projection model

In this configuration, a point $P_{i}$ and a line $l_{j}$ on the object coordinates are projected onto a point $Q_{i}$ and a line $L_{j}$ respectively in the normalized image plane. The point $Q_{i}$ in the imaging coordinates $(u, v)$ is given by equations (8) and (9).

$$
\begin{aligned}
& u_{i}=\frac{\vec{i} \cdot \vec{P}_{i}+t_{x}}{\vec{k} \cdot \vec{P}_{i}+t_{z}}=\frac{\vec{I} \cdot \vec{P}_{i}+u_{0}}{\varepsilon_{i}+1} \\
& v_{i}=\frac{\vec{j} \cdot \vec{P}_{i}+t_{y}}{\vec{k} \cdot \vec{P}_{i}+t_{z}}=\frac{\vec{J} \cdot \vec{P}_{i}+v_{0}}{\varepsilon_{i}+1} \\
& \vec{I}=\vec{i} / t_{z}, \vec{J}=\vec{j} / t_{z}, \varepsilon_{i}=\vec{k} \cdot \vec{P}_{i} / t_{z}
\end{aligned}
$$

where $\vec{P}_{i}$ is a position vector of a point $P_{i}, \vec{i}, \vec{j}, \vec{k}$ are the unit vectors of $x, y, z$ axes, respectively for the object coordinates and $\left(u_{0}, v_{0}\right)=\left(t_{x} / t_{z}, t_{y} / t_{z}\right)$.

And a line $l_{j}$ on the object coordinates, which is expressed by equation (10), is also imaged as a line $L_{j}$ in virtual plane as a line equation (11).

$$
l_{j}: \overrightarrow{P_{j}}=\overrightarrow{W_{j}}+\lambda \cdot \overrightarrow{V_{j}} \quad \lambda \in \mathbb{R}^{1}
$$

where $\vec{V}_{j} \in \mathbb{R}^{3 \times 1}$ is a directional vector of the line $l_{j}$ and $\vec{W}_{j} \in \mathbb{R}^{3 \times 1}$ is a point on the line 


$$
L_{j}: a_{j}\left(\vec{I} \cdot \overrightarrow{P_{j}}+u_{0}\right)+b_{j}\left(\vec{J} \cdot \overrightarrow{P_{j}}+v_{0}\right)+c_{j}\left(1+\varepsilon_{i}\right)=0
$$

\subsection{Pose estimation by para-perspective method}

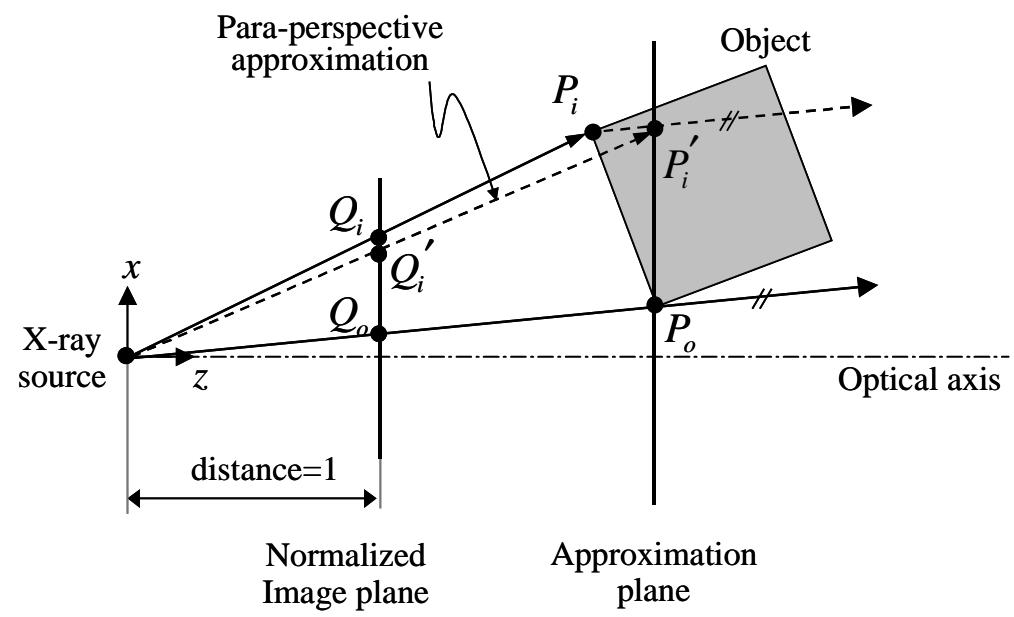

Figure 9. Para-perspective projection

In this research, pose estimation is performed by using a para-perspective iterative method that was introduced by Dornaika and Garcia [8]. Para-perspective approximation is illustrated in figure 9, where it is explained in 2D space for convenience. Here, it is approximated that an object point $\mathrm{P}_{\mathrm{i}}$ is imaged at a point $Q_{i}^{\prime}$ rather than its real projection point $Q_{i}$ on the normalized image plane. As shown in the figure, the projection follows a parallel ray approximation, where all the points on the object coordinates lie in the approximated plane via rays parallel to the ray passing through the origin of the object coordinates. This para-perspective model is mathematically achieved by a first-order approximation of $1 /\left(1+\varepsilon_{i}\right)$ as in equation (12) in the perspective model:

$$
\frac{1}{1+\varepsilon_{i}} \approx 1-\varepsilon_{i}, i \in\{1 \ldots n\}
$$

By applying this approximation to equations (8), we obtain a para-perspective approximated projection $Q_{i}^{p}\left(u_{i}^{p}, v_{i}^{p}\right)$ :

$$
\begin{aligned}
& u_{i}^{p}=\left(\vec{I} \cdot \vec{P}_{i}+u_{0}\right)\left(1-\varepsilon_{i}\right) \approx \vec{I} \cdot \vec{P}_{i}+u_{0}-u_{0} \varepsilon_{i} \\
& v_{i}^{p}=\left(\vec{J} \cdot \vec{P}_{i}+v_{0}\right)\left(1-\varepsilon_{i}\right) \approx \vec{J} \cdot \vec{P}_{i}+v_{0}-v_{0} \varepsilon_{i} .
\end{aligned}
$$

Then, the relation between the para-perspective $\left(x_{i}^{p}, y_{i}^{p}\right)$ and the perspective projection $(x, y)$ of $P_{i}$ in the normalized plane is written by :

$$
\begin{aligned}
& u_{i}^{p}=u_{i}\left(1+\varepsilon_{i}\right)-u_{0} \varepsilon_{i} \\
& v_{i}^{p}=v_{i}\left(1+\varepsilon_{i}\right)-v_{0} \varepsilon_{i} .
\end{aligned}
$$

The para-perspective equation (14) is rewritten as equation (15) including the parameters of the pose : 


$$
\begin{aligned}
& u_{i}^{p}-u_{0}=\frac{\vec{i}-u_{0} \vec{k}}{t_{z}} \vec{P}_{i} \\
& v_{i}^{p}-v_{0}=\frac{\vec{j}-v_{0} \vec{k}}{t_{z}} \vec{P}_{i} .
\end{aligned}
$$

By substituting equation (8) and (13) in equations (15), we obtain :

$$
\begin{aligned}
& \left(u_{i}-u_{0}\right)\left(1+\varepsilon_{i}\right)=\overrightarrow{P_{i}} \cdot \overrightarrow{I_{p}} \\
& \left(v_{i}-v_{0}\right)\left(1+\varepsilon_{i}\right)=\vec{P}_{i} \cdot \overrightarrow{J_{p}}
\end{aligned}
$$

where $\vec{I}_{p}=\frac{\vec{i}-u_{0} \vec{k}}{t_{z}}$ and $\overrightarrow{J_{p}}=\frac{\vec{j}-v_{0} \vec{k}}{t_{z}}$.

In this manner, a line on the normalized plane (11) can also be rewritten as

$$
\begin{aligned}
& a_{j} \vec{W}_{j} \cdot \vec{I}_{p}+b_{j} \vec{W}_{j} \cdot \vec{J}_{p}+\left(a_{j} u_{0}+b_{j} v_{0}+c_{j}\right)\left(1+\eta_{j}\right)=0 \\
& a_{j} \vec{V}_{j} \cdot \vec{I}_{p}+b_{j} \vec{V}_{j} \cdot \vec{J}_{p}+\left(a_{j} u_{0}+b_{j} v_{0}+c_{j}\right) \xi_{j}=0
\end{aligned}
$$

where $\eta_{j}=\vec{k} \cdot \overrightarrow{W_{j}} / t_{z}, \xi_{j}=\vec{k} \cdot \overrightarrow{V_{j}} / t_{z}$.

As a result, two constraint equations to determine the pose transformation vectors $\vec{I}_{p}, \vec{J}_{p}$ are provided by equation (16) on each point feature, and another two constraint equations can be prepared in equation (17) from a line feature in an image if it is available. In case of a polyhedral object, a number of corner points and lines are available as the features used in determining the vectors $\vec{I}_{p}, \vec{J}_{p}$. When $n$ corner points and $m$ lines are detected in the image, $2(n+m)$ equations are available and written as a matrix form:

$$
\mathbf{G} \cdot\left[\begin{array}{l}
\vec{I}_{p} \\
\vec{J}_{p}
\end{array}\right]=\mathbf{C} \quad ; \quad \mathbf{G} \in \mathbb{R}^{2(m+n) \times 6}, \mathbf{C} \in \mathbb{R}^{2(m+n) \times 1}
$$

where the matrices $\mathbf{G}$ and $\mathbf{C}$ are calculated by the image features through equation (19).

$$
\mathbf{G}=\left[\begin{array}{cc}
P_{1}^{T} & 0^{T} \\
0^{T} & P_{1}^{T} \\
\vdots & \vdots \\
P_{m}^{T} & 0^{T} \\
0^{T} & P_{m}^{T} \\
a_{1} W_{1}^{T} & b_{1} W_{1}^{T} \\
a_{1} V_{1}^{T} & b_{1} V_{1}^{T} \\
\vdots & \vdots \\
a_{n} W_{n}^{T} & b_{n} W_{n}^{T} \\
a_{n} V_{n}^{T} & b_{n} V_{n}^{T}
\end{array}\right], \mathbf{C}=\left[\begin{array}{c}
\left(u_{1}-u_{0}\right)\left(1+\varepsilon_{1}\right) \\
\left(v_{1}-v_{0}\right)\left(1+\varepsilon_{1}\right) \\
\vdots \\
\left(u_{m}-u_{0}\right)\left(1+\varepsilon_{m}\right) \\
\left(v_{m}-v_{0}\right)\left(1+\varepsilon_{m}\right) \\
-\left(a_{1} u_{0}+b_{1} v_{0}+c_{1}\right)\left(1+\eta_{1}\right) \\
-\left(a_{1} u_{0}+b_{1} v_{0}+c_{1}\right) \xi_{1} \\
\vdots \\
-\left(a_{n} u_{0}+b_{n} v_{0}+c_{n}\right)\left(1+\eta_{n}\right) \\
-\left(a_{n} u_{0}+b_{n} v_{0}+c_{n}\right) \xi_{n}
\end{array}\right] .
$$

Finally, the pose of an object is estimated by solving the equation (18) and determining the transformation vector $\left[\vec{I}_{p}, \vec{J}_{p}\right]^{T}$. The estimation is performed iteratively, since the equation (18) comes from a simplified para-perspective model and the 
equation itself includes estimation error.

The procedure of the iteration is summarized as :

Step 1. Para-perspective estimation :

To initialize para-perspective parameters $\varepsilon_{i}=0, \eta_{j}=0, \xi_{\mathrm{j}}=0 \quad ; i=1,2, \ldots, n$ and $j=1,2, \ldots, m$.

Step 2. To calculate $\left[\vec{I}_{p} \vec{J}_{p}\right]^{T}=(\mathbf{G G})^{-1} \mathbf{G}^{\mathrm{T}} \mathbf{C}$

Step 3. To calculate pose parameters $\mathbf{R}, \mathbf{T}$ using $\left[\vec{I}_{p}, \vec{J}_{p}\right]^{T}$

$$
\begin{aligned}
& \mathbf{T}=\left[t_{x}, t_{y}, t_{z}\right]^{T} ; t_{z}=\frac{1}{2}\left(\frac{\sqrt{1+u_{0}^{2}}}{\left\|\vec{I}_{p}\right\|}+\frac{\sqrt{1+v_{0}^{2}}}{\left\|\vec{J}_{p}\right\|}\right), t_{x}=u_{0} t_{z} \text { and } t_{y}=v_{0} t_{z} \\
& \mathbf{R}=\left[\begin{array}{lll}
\vec{i} & \vec{j} & \vec{k}
\end{array}\right]^{T} \\
& \vec{k}=t_{z}^{2} \cdot \vec{I}_{p} \times \vec{J}_{p}+t_{z} \cdot\left(v_{0} \vec{I}_{p} \times \vec{k}-u_{0} \vec{J}_{p} \times \vec{k}\right) \\
& \vec{i}=t_{z} \vec{I}_{p}+u_{0} \vec{k}, \vec{j}=t_{z} \vec{J}_{p}+v_{0} \vec{k}
\end{aligned}
$$

Step 4. To update the para-perspective parameters

$$
\varepsilon_{i}=\frac{\vec{k} \cdot \vec{P}_{i}}{t_{z}}, \eta_{j}=\frac{\vec{k} \cdot \vec{W}_{j}}{t_{z}}, \xi_{\mathrm{j}}=\frac{\vec{k} \cdot \vec{V}_{j}}{t_{z}}
$$

Step 5. To go to step 2 and continue the procedure until there is no meaningful change in the para-perspective parameters.

\section{SIMULATION RESULTS}

We conducted a series of simulations on three different sample objects, which are a cube, a pyramid and an octahedral object of which x-ray images are shown in figure 11. Table 2 represents the simulation results for these three objects, where the line features are mainly used in the estimation and corner points are partially used. The simulations are conducted on 55 different poses for each object, and the averaged errors of the results are listed in the table. From the results, it can be pointed out that as the number of features increases in the estimation, the estimation error is decreased. In most cases, the estimation is finished within 3 iterations. However, there is an important fact that the number of iteration for convergence is related to the distance between an object and x-ray source due to the para-perspective approximation error. Since the paraperspective model is a parallel ray approximation, thus has a large approximation error when an object is located near to the $\mathrm{X}$-ray source. Figure 11 shows the trend of reduced iterations according to the distance between an object and x-ray source. For convenience, the distance is normalized with the representative size of an object. As shown in this figure, the iteration number for a successful estimation is reduced from 19 to 3 as the normalized distance varies from 0.8 to 2 . And it does not converged any more when the normalized distance is less than 0.8 .

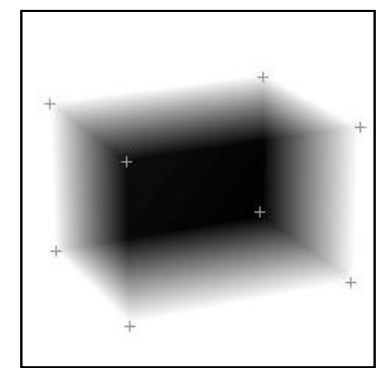

(a) cube

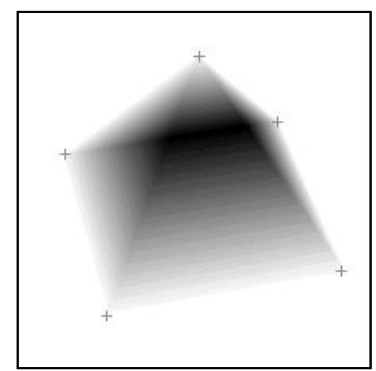

(b) pyramid

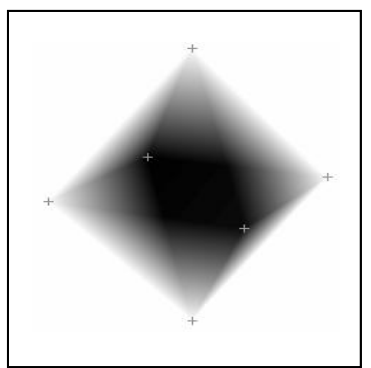

(c) octahedron

Figure 10. The simulation images of polyhedral objects 
Table 2. The error of pose estimation

(a) Results for a cube

\begin{tabular}{|c|c|c|c|}
\hline & \multicolumn{3}{|c|}{ paraperspective iteration } \\
\hline $\begin{array}{c}\text { No. of } \\
\text { correspondences }\end{array}$ & $\begin{array}{l}\text { Rot. Error } \\
\text { (deg.) }\end{array}$ & $\begin{array}{c}\text { Trans. Error } \\
(\%)\end{array}$ & $\begin{array}{l}\text { No. of } \\
\text { iterations }\end{array}$ \\
\hline 12 lines +1 point & 0.33 & 0.45 & 2 \\
\hline 12 lines +2 points & 0.31 & 0.32 & 2 \\
\hline 12 lines +3 points & 0.31 & 0.28 & 3 \\
\hline 12 lines +4 points & 0.28 & 0.25 & 2 \\
\hline 12 lines +5 points & 0.25 & 0.20 & 2 \\
\hline 12 lines +6 points & 0.23 & 0.20 & 2 \\
\hline 12 lines +7 points & 0.21 & 0.20 & 2 \\
\hline 12 lines +8 points & 0.21 & 0.19 & 2 \\
\hline average & 0.27 & 0.26 & 2 \\
\hline
\end{tabular}

(b) Results for a pyramid

\begin{tabular}{|c|c|c|c|}
\hline & \multicolumn{3}{|c|}{ paraperspective iteration } \\
\hline $\begin{array}{c}\text { No. of } \\
\text { correspondences }\end{array}$ & $\begin{array}{c}\text { Rot. Error } \\
\text { (deg.) }\end{array}$ & $\begin{array}{c}\text { Trans. Error } \\
(\%)\end{array}$ & $\begin{array}{c}\text { No. of } \\
\text { iterations }\end{array}$ \\
\hline 8 lines + 1 point & 0.48 & 0.29 & 3 \\
8 lines + 2 points & 0.52 & 0.45 & 2 \\
8 lines + 3 points & 0.53 & 0.38 & 2 \\
8 lines + 4 points & 0.51 & 0.29 & 2 \\
8 lines + 5 points & 0.43 & 0.26 & 2 \\
\hline average & 0.50 & 0.33 & 2 \\
\hline
\end{tabular}

(c) Results for an octahedral object

\begin{tabular}{|c|c|c|c|}
\hline & \multicolumn{3}{|c|}{ paraperspective iteration } \\
\hline $\begin{array}{c}\text { No. of } \\
\text { correspondences }\end{array}$ & $\begin{array}{c}\text { Rot. Error } \\
\text { (deg.) }\end{array}$ & $\begin{array}{c}\text { Trans. Error } \\
(\%)\end{array}$ & $\begin{array}{c}\text { No. of } \\
\text { iterations }\end{array}$ \\
\hline 12 lines + 1 point & 0.54 & 0.49 & 2 \\
12 lines + 2 points & 0.52 & 0.50 & 2 \\
12 lines + 3 points & 0.51 & 0.41 & 2 \\
12 lines + 4 points & 0.48 & 0.41 & 2 \\
12 lines + 5 points & 0.44 & 0.43 & 2 \\
12 lines + 6 points & 0.43 & 0.40 & 2 \\
\hline average & 0.49 & 0.44 & 2 \\
\hline
\end{tabular}

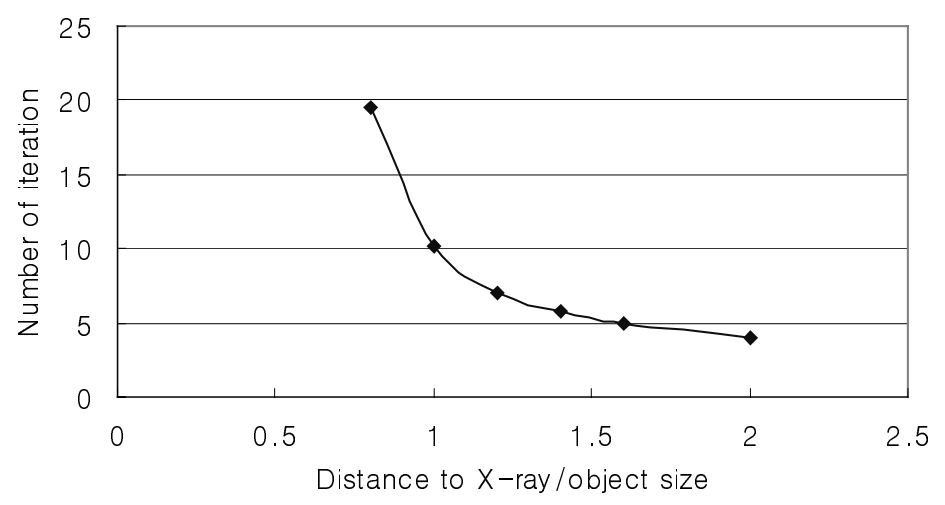

Figure 11. Iteration numbers according to the normalized distance

\section{CONCLUSION}

In this paper, a pose estimation for a polyhedral object using an x-ray image has been studied, which is based on the image features of a polyhedral object such as corner points and line edges. To achieve this, we proposed a series of image processing methods, which includes an edge detection algorithm named as a plane fit method, to detect features efficiently in $\mathrm{x}$-ray images. From the simulations, the corner points are detected successfully with the averaged error about 0.8 pixel.

From the features of lines and the corner points detected in x-ray image, the pose of an object can be estimated iteratively using the para-perspective approximation method. The estimations are performed on images of various poses, and the error is less than 0.5 (degree) and $0.44(\%)$ in rotation and translation respectively. The iteration for the successful estimation was related to the distance between an object and $\mathrm{x}$-ray source due to the para-perspective approximation error. Once the normalized distance is beyond 0.8 , in our simulations, the iterative estimation converged successfully with sufficiently small errors. In this research, only polynomial objects were considered for the convenience of defining image features, however, it needs to be expanded to the generalized ones including curved surfaces such as a cylinder, a cone and so on. 


\section{ACKNOWLEDGMENTS}

This work was supported by Pohang Steel Compony as an industrial project "three dimensional volume measurement of an object using x-ray images".

\section{REFERENCES}

1. S. Lavallee, R. Szeliske, "Recovering the Position and Orientation of Free-Form Objects from Image Contours Using 3D Distance Maps", IEEE transactions on pattern analysis and machine intelligence, vol. 17, no 4, pp. 378-390, April 1995.

2. D. G. Lowe, "Fitting parameterized three-dimensional models to images", , IEEE transactions on pattern analysis and machine intelligence, vol. 13, no. 5, pp. 441-450, May 1996.

3. W. A. Hoff, R. D. Komistek, D. A. Dennis, S. Walker, E. Northcut, K. Spargo, "Pose Estimation of Artificial Knee Implants in Fluoroscopy Images Using A Template Matching Technique”, IEEE, 1996, pp181-186

4. "Computed tomography details casting defects", Advanced Materials \& Process, Nov. 1990, pp 54-66.

5. Y. Ohta, K. Maenobu, T. Sakai, "Obtaining surface orientation from texels under perspective projection", Proceedings of the $7^{\text {th }}$ IJCAI, 1981.

6. R. Jain, R. Kasturi, B. G. Schunck, “Machine Vision”, McGraw-Hill, 1995, pp 140-157

7. D. H. Ballard, C. M. Brown, "Computer Vision", Prentice-Hall, 1982, pp 83-84

8. F. Dornaika, C. Garcia, "Pose Estimation using Point and Line Correspondences”, Real-Time Images 5, 1999, pp 215230

9. Y. K. Ryu and H. S. Cho, "Visual inspection scheme for use in optical solder joint inspection system", IEEE ICRA '96, Mennesota, USA, 1996

10. Y. J. Roh, "The Analysis and Design of x-ray Cross Sectional Imaging System Using Digital Tomosynthesis", M.S. thesis, KAIST, 1997

11. T. S. Kang, K. W. Ko, W. S. Park, Y. J. Roh, H. S. Cho, "PCB joint inspection using X-ray", teck. report LCA-9802-V3/8, LCA, KAIST, 1998

12. T. S. Kang, K. W. Ko, W. S. Park, Y. J. Roh, H. S. Cho, "PCB joint inspection using X-ray", teck. report LCA-9612-V2/5, LCA, KAIST, 1996 